\title{
Stochastic inference of surface-induced effects using Brownian motion
}

\author{
Maxime Lavaud $\odot,^{1}$ Thomas Salez $\odot{ }^{1,2, *}$ Yann Louyer, ${ }^{1}$ and Yacine Amarouchene ${ }^{1}{ }^{1, \dagger}$ \\ ${ }^{1}$ Univ. Bordeaux, CNRS, LOMA, UMR 5798, F-33405 Talence, France \\ ${ }^{2}$ Global Station for Soft Matter, Global Institution for Collaborative Research and Education, \\ Hokkaido University, Sapporo, Hokkaido 060-0808, Japan
}

(Received 8 December 2020; revised 28 April 2021; accepted 2 June 2021; published 8 July 2021)

\begin{abstract}
Brownian motion in confinement and at interfaces is a canonical situation, encountered from fundamental biophysics to nanoscale engineering. Using the Lorenz-Mie framework, we optically record the thermally induced tridimensional trajectories of individual microparticles, within salty aqueous solutions, in the vicinity of a rigid wall, and in the presence of surface charges. We construct the time-dependent position and displacement probability density functions, and study the non-Gaussian character of the latter which is a direct signature of the hindered mobility near the wall. Based on these distributions, we implement a robust and self-calibrated multifitting method, allowing for the thermal-noise-limited inference of diffusion coefficients spatially resolved at the nanoscale, equilibrium potentials, and forces at the femtonewton resolution.
\end{abstract}

DOI: 10.1103/PhysRevResearch.3.L032011

Brownian motion is a central paradigm in modern science. It has implications in fundamental physics, biology, and even finance, to name a few. By understanding that the apparent erratic motion of colloids is a direct consequence of the thermal motion of surrounding fluid molecules, pioneers like Einstein and Perrin provided decisive evidence for the existence of atoms $[1,2]$. Specifically, free Brownian motion in the bulk is characterized by a typical spatial extent evolving as the square root of time, as well as Gaussian displacements.

At a time of miniaturization and interfacial science, and moving beyond the idealized bulk picture, it is relevant to consider the added roles of boundaries to the above context. Indeed, Brownian motion at interfaces and in confinement is a widespread practical situation in microbiology and nanofluidics. In such a case, surface effects become dominant and alter drastically the Brownian statistics, with key implications towards (i) the understanding and smart control of the interfacial dynamics of microscale entities and (ii) high-resolution measurements of surface forces at equilibrium. Interestingly, a confined colloid will exhibit non-Gaussian statistics in displacements, due to the presence of multiplicative noises induced by the hindered mobility near the wall [3-5]. Additionally, the particle can be subjected to electrostatic or van der Waals forces [6] exerted by the interface, and might experience slippage too $[7,8]$. Considering the two-body problem, the nearby boundary can also induce some effective interaction [9]. Previous studies have designed novel methods to

\footnotetext{
*thomas.salez@u-bordeaux.fr

†yacine.amarouchene@u-bordeaux.fr

Published by the American Physical Society under the terms of the Creative Commons Attribution 4.0 International license. Further distribution of this work must maintain attribution to the author(s) and the published article's title, journal citation, and DOI.
}

measure the diffusion coefficient of confined colloids [10-16], or to infer surface forces [17-22]. However, such a statistical inference is still an experimental challenge, and a precise calibration-free method taking simultaneously into account the whole ensemble of relevant properties, over broad spatial and time ranges, is currently lacking.

In this Rapid Communication, we aim at filling the previously identified gap by implementing a method of statistical inference on a set of trajectories of individual microparticles recorded by holographic microscopy. The buoyant particles are free to evolve within salty aqueous solutions, near a rigid substrate, and in the presence of surface charges. We primarily reconstruct the equilibrium probability distribution function of the position, as well as the time-resolved probability distribution functions of the displacements in directions transverse and normal to the wall, including in particular the meansquared displacements (MSDs). Special attention is dedicated to the non-Gaussian statistics, for time scales broadly ranging from tens of milliseconds to several tens of minutes. Furthermore, we implement the advanced inference method recently proposed [23]. Additionally, an optimization scheme is used in order to determine precisely all the free physical parameters and the actual distance to the wall, at once. All together, this procedure leads to the robust calibration-free inference of the two central quantities of the problem: (i) the space-dependent short-term diffusion coefficients, with a nanoscale spatial resolution; and (ii) the total force experienced by the particle, at the thermal-noise limited femtonewton resolution. These main results are summarized in Fig. 1, the goal of this Rapid Communication being the detailed obtention of which.

The experimental setup is schematized in Fig. 2(a). A sample consists of a parallelepipedic chamber $(1.5 \mathrm{~cm} \times$ $1.5 \mathrm{~cm} \times 150 \mu \mathrm{m})$, made from two glass covers and a parafilm spacer and sealed with vacuum grease, containing a dilute suspension of spherical polystyrene beads (Sigma Aldrich) with nominal radii $a=1.5 \pm 0.035 \mu \mathrm{m}$, at room 

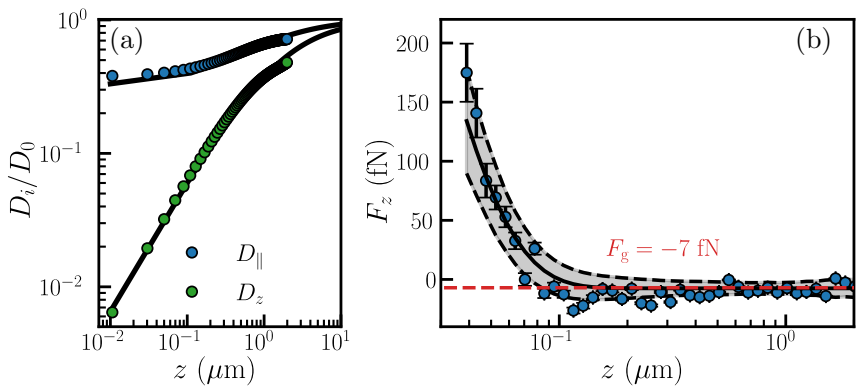

FIG. 1. (a) Measured local short-term diffusion coefficients $D_{i}$ of the microparticle, normalized by the bulk value $D_{0}$, as functions of the distance $z$ to the wall [see Fig. 2(c)], along both a transverse direction $x$ or $y\left(D_{i}=D_{\|}=D_{x}=D_{y}\right.$, blue $)$ and the normal direction $z\left(D_{i}=D_{z}\right.$, green) to the wall. The solid lines are the theoretical predictions, $D_{\|}(z)=D_{0} \eta / \eta_{\|}(z)$ and $D_{z}(z)=D_{0} \eta / \eta_{z}(z)$, using the local effective viscosities $\eta_{\|}(z)$ and $\eta_{z}(z)$ of Eqs. (3) and (4), respectively. (b) Total normal conservative force $F_{z}$ exerted on the particle as a function of the distance $z$ to the wall, reconstructed from Eq. (11), using Eq. (4). The solid line corresponds to Eq. (13), with $B=4.8$, $\ell_{\mathrm{D}}=21 \mathrm{~nm}$, and $\ell_{\mathrm{B}}=530 \mathrm{~nm}$. The black dashed lines and gray area indicate the amplitude of the thermal noise computed from Eq. (12). The horizontal red dashed line indicates the buoyant weight $F_{\mathrm{g}}=-7$ $\mathrm{fN}$ of the particle.

temperature $T$, in distilled water (type 1, MilliQ device) of viscosity $\eta=1 \mathrm{mPa}$ s. The sample is illuminated by a collimated laser beam with a $532-\mu \mathrm{m}$ wavelength. The light scattered by one colloidal particle at a given time $t$ interferes with the incident beam. An oil-immersion objective lens (x60 magnification, 1.30 numerical aperture) collects the resulting instantaneous interference pattern, and relays it to a camera
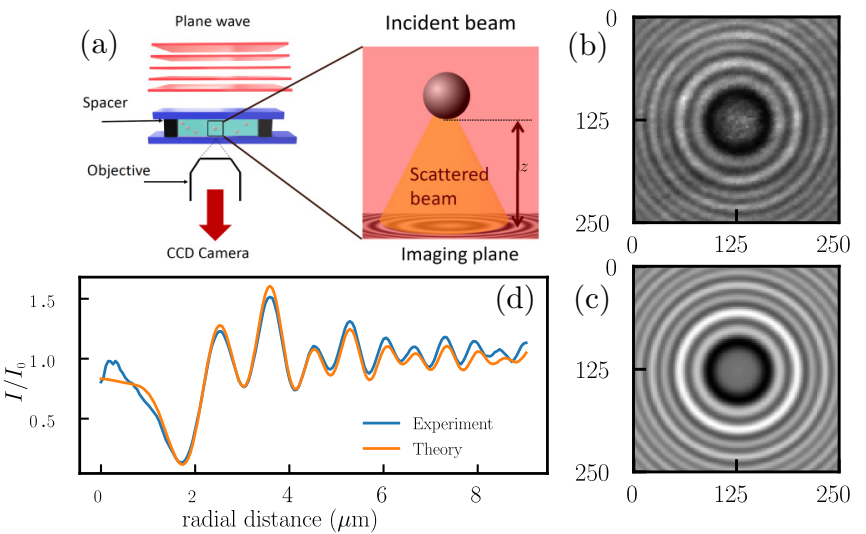

FIG. 2. (a) Schematic of the experimental setup. A laser plane wave of intensity $I_{0}$ illuminates the chamber containing a dilute suspension of microspheres in water. The light scattered by a particle interferes with the incident beam onto the focal plane of an objective lens, that magnifies the interference pattern and relays it to a camera. (b) Typical experimental interference pattern produced by one particle. (c) Corresponding best-fit Lorenz-Mie interference pattern [24-28], providing a distance $z=11.24 \pm 0.2 \mu \mathrm{m}$ to the wall, as well as the radius $a=1.518 \pm 0.006 \mu \mathrm{m}$ and refractive index $n=1.584 \pm 0.006$ of the particle. (d) Angular averages of the intensities $I$ (normalized by $I_{0}$ ) from the experimental and theoretical interference patterns, as functions of the radial distance to the $z$ axis. (a)
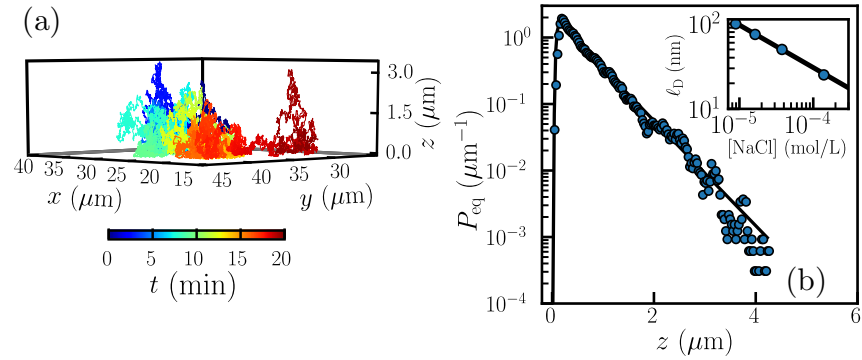

FIG. 3. (a) Typical measured tridimensional trajectory $\mathbf{r}(\mathbf{t})=$ $[\mathbf{x}(\mathbf{t}), \mathbf{y}(\mathbf{t}), \mathbf{z}(\mathbf{t})]$ of the microparticle near the wall $(z=0)$. (b) Measured equilibrium probability density function $P_{\mathrm{eq}}$ of the distance $z$ between the particle and the wall. The solid line represents the best fit to the normalized Gibbs-Boltzmann distribution in position, using the total potential energy $U(z)$ of Eq. (1), with $B=4.8, \ell_{\mathrm{D}}=21 \mathrm{~nm}$, and $\ell_{\mathrm{B}}=530 \mathrm{~nm}$. The inset shows the measured Debye length $\ell_{\mathrm{D}}$ as a function of salt concentration $[\mathrm{NaCl}]$. The solid line is the expected Debye relation $\ell_{\mathrm{D}}=0.304 / \sqrt{[\mathrm{NaCl}]}$, for a single monovalent salt in water at room temperature.

with a 51.6-nm/pixel resolution [see Fig. 2(b)]. The exposure time for each frame is fixed to $3 \mathrm{~ms}$ to avoid motion-induced blurring of the image. The angular average of the intensity profile from each time frame is then fitted [see Figs. 2(c) and 2(d)] to the Lorenz-Mie scattering function [24-28], which provides the particle radius $a$, its refractive index $n$, and its instantaneous tridimensional position $\mathbf{r}=(\mathbf{x}, \mathbf{y}, \mathbf{z})$. To reduce the uncertainty on the position measurement, we first calibrate $a=1.518 \pm 0.006 \mu \mathrm{m}$ and $n=1.584 \pm 0.006 \mathrm{sep}$ arately from the first $10^{5}$ time frames. The obtained refractive index is consistent with the one reported in [16]. Then, for each subsequent time frame, the only remaining fitted quantity is $\mathbf{r}$, which allows us to reconstruct the trajectory $\mathbf{r}(t)$ with a nanometric spatial resolution, as shown in Fig. 3(a).

Using the trajectory of the particle, one can then construct the equilibrium probability density function $P_{\mathrm{eq}}(\mathbf{r})$ of the position of the particle. We find that it does not depend on $x$ and $y$, but only on the distance $z$ between the particle and the wall. As seen in Fig. 3(b), an exponential tail is observed at large distance, which is identified to the sedimentation contribution in Perrin's experiment [2], but here with the probability density function of a single particle instead of the concentration field. In contrast, near the wall, we observe an abrupt depletion, indicating a repulsive electrostatic contribution. Indeed, when immersed in water, both the glass substrate and the polystyrene bead are negatively charged. All together, the total potential energy $U(z)$ thus reads

$$
\frac{U(z)}{k_{\mathrm{B}} T}=\left\{\begin{array}{l}
B e^{-\frac{z}{\ell_{\mathrm{D}}}}+\frac{z}{\ell_{\mathrm{B}}}, \quad \text { for } z>0 \\
+\infty, \quad \text { for } z \leqslant 0
\end{array},\right.
$$

where $k_{\mathrm{B}}$ is the Boltzmann constant, $B$ is a dimensionless number related to the surface electrostatic potentials of the particle and the wall [17], $\ell_{\mathrm{D}}$ is the Debye length, $\ell_{\mathrm{B}}=k_{\mathrm{B}} T /(g \Delta m)$ is the Boltzmann length, $g$ is the gravitational acceleration, and $\Delta m$ is the (positive) buoyant mass of the particle. From this total potential energy, one can then construct the Gibbs-Boltzmann distribution $P_{\text {eq }}(z)=$ $A \exp \left[-U(z) /\left(k_{\mathrm{B}} T\right)\right]$ in position, where $A$ is a normalization 
constant, that fits the data very well, as shown in Fig. 3(b). Moreover, as shown in the inset of Fig. 3(b), we verified that we recover the Debye relation $\ell_{\mathrm{D}}=0.304 / \sqrt{[\mathrm{NaCl}]}$, with $\ell_{\mathrm{D}}$ in $\mathrm{nm}$, and where $[\mathrm{NaCl}]$ is the concentration of salt in $\mathrm{mol} / \mathrm{L}$, with a prefactor corresponding to a single monovalent salt in water at room temperature [29]. Besides, we have verified (not shown) that the dimensionless parameter $B=4.8$ related to surface charges is constant in the studied salt-concentration range, thus excluding any nonlinear effect $[21,30]$ in our case.

We now turn to dynamical aspects, by considering the MSD. For the three spatial directions, indexed by $i=x, y$, and $z$, corresponding to the coordinates $r_{x}=x, r_{y}=y$, and $r_{z}=z$, of the position $\mathbf{r}$, and for a given time increment $\Delta t$, the MSD is defined as

$$
\left\langle\Delta r_{i}(t)^{2}\right\rangle_{t}=\left\langle\left[r_{i}(t+\Delta t)-r_{i}(t)\right]^{2}\right\rangle_{t},
$$

where the average \langle\rangle$_{t}$ is performed over time $t$. For a free Brownian motion in the bulk, and in the absence of other forces than the dissipative and random ones, the MSD is linear in time, i.e., $\left\langle\Delta r_{i}(t)^{2}\right\rangle_{t}=2 D_{0} \Delta t$, where $D_{0}=k_{\mathrm{B}} T /(6 \pi \eta a)$ is the bulk diffusion coefficient given by the Stokes-Einstein relation [1], and $\eta$ is the liquid viscosity. Further including sedimentation restricts the validity of the previous result along $z$ to short times only, i.e., for $\Delta t \ll \ell_{\mathrm{B}}^{2} / D_{0}$ such that the vertical diffusion is not yet affected by the gravitational drift.

The presence of a rigid wall at $z=0$ adds a repulsive electrostatic force along $z$. It also decreases the mobilities nearby through hydrodynamic interactions, leading to effective viscosities $\eta_{\|}(z)=\eta_{x}(z)=\eta_{y}(z)$, and $\eta_{z}(z)$. The latter are [31]

$$
\eta_{\|}=\frac{\eta}{1-\frac{9}{16} \xi+\frac{1}{8} \xi^{3}-\frac{45}{256} \xi^{4}-\frac{1}{16} \xi^{5}},
$$

where $\xi=a /(z+a)$, and

$$
\eta_{z}=\eta \frac{6 z^{2}+9 a z+2 a^{2}}{6 z^{2}+2 a z},
$$

which is Padé approximated within $1 \%$ accuracy [32].

Interestingly, despite the previous modifications, the temporal linearity of the MSD is not altered by the presence of the wall $[17,33]$ for $x$ and $y$, as well as at short times for $z$. In such cases, the MSD reads

$$
\left\langle\Delta r_{i}(t)^{2}\right\rangle_{t}=2\left\langle D_{i}\right\rangle \Delta t
$$

where for each spatial direction we introduced the local diffusion coefficient $D_{i}(z)=D_{0} \eta / \eta_{i}(z)$, and its average $\left\langle D_{i}(z)\right\rangle=$ $\int_{0}^{\infty} d z D_{i}(z) P_{\mathrm{eq}}(z)$ against the Gibbs-Boltzmann distribution in position. As shown in Fig. 4(a), the MSD measured along $x$ or $y$ is indeed linear in time. By fitting to Eq. (5), using Eqs. (1) and (3), we extract an average transverse diffusion coefficient $\left\langle D_{\|}\right\rangle=\left\langle D_{x}\right\rangle=\left\langle D_{y}\right\rangle=0.52 D_{0}$. In contrast, along $z$, we identify two different regimes: one at short times, where the MSD is still linear in time, with a similarly obtained best-fit value of $\left\langle D_{z}\right\rangle=0.24 D_{0}$; and one at long times, where the MSD saturates to a plateau. This latter behavior indicates that the equilibrium regime has been reached, with the particle having essentially explored all the relevant positions given by the Gibbs-Boltzmann distribution.

Having focused on the MSD, i.e., on the second moment only, we now turn to the full probability density function
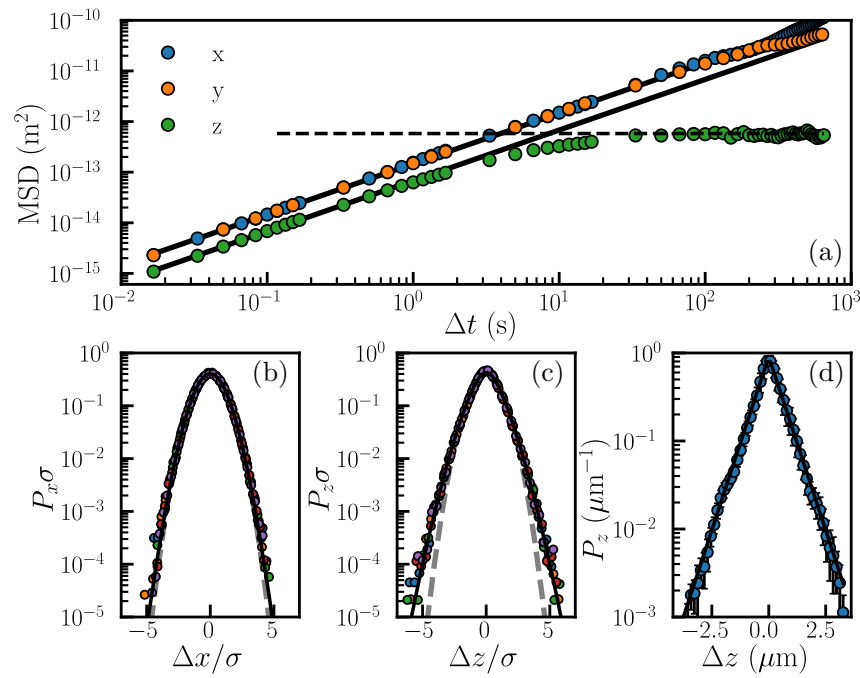

FIG. 4. (a) Measured mean-squared displacements [MSD, see Eq. (2)] as functions of the time increment $\Delta t$, for the three spatial directions, $x, y$, and $z$. The solid lines are best fits to Eq. (5), using Eqs. (1), (3), and (4), with $B=4.8, \ell_{\mathrm{D}}=21 \mathrm{~nm}$, and $\ell_{\mathrm{B}}=530 \mathrm{~nm}$, providing the average diffusion coefficients $\left\langle D_{\|}\right\rangle=\left\langle D_{x}\right\rangle=\left\langle D_{y}\right\rangle=$ $0.52 D_{0}$ and $\left\langle D_{z}\right\rangle=0.24 D_{0}$. The dashed line is the best fit to Eq. (8), using Eq. (1), with $B=4.8, \ell_{\mathrm{D}}=21 \mathrm{~nm}$, and $\ell_{\mathrm{B}}=530 \mathrm{~nm}$. (b, c) Normalized probability density functions $P_{i} \sigma$ of the normalized displacements $\Delta x / \sigma$ and $\Delta z / \sigma$, at short times, with $\sigma^{2}$ the corresponding MSD [see panel (a)], for different time increments $\Delta t$ ranging from 0.0167 to $0.083 \mathrm{~s}$, as indicated with different colors. The solid lines are the best fits to Eq. (6), using Eqs. (1), (3), and (4), with $B=4.8, \ell_{\mathrm{D}}=21 \mathrm{~nm}$, and $\ell_{\mathrm{B}}=530 \mathrm{~nm}$. For comparison, the gray dashed lines are normalized Gaussian distributions, with zero means and unit variances. (d) Probability density function $P_{z}$ of the displacement $\Delta z$, at long times, averaged over several values of $\Delta t$ ranging between 25 and $30 \mathrm{~s}$. The solid line is the best fit to Eq. (7), using Eq. (1), with $B=4.8, \ell_{\mathrm{D}}=21 \mathrm{~nm}$, and $\ell_{\mathrm{B}}=530 \mathrm{~nm}$.

$P_{i}$ of the displacement $\Delta r_{i}$. Since, the diffusion coefficient $D_{i}(z)$ varies as a result of the variation of $z$ along the particle trajectory, $P_{i}$ exhibits a non-Gaussian behavior, as seen in Figs. 4(b)-4(d). We stress that we even resolve the onset of a non-Gaussian behavior in $P_{x}$, by zooming on the large- $|\Delta x|$ wings (not shown). At short times, $P_{i}$ can be modeled by the averaged diffusion Green's function [16,34]:

$$
P_{i}\left(\Delta r_{i}\right)=\int_{0}^{\infty} d z P_{\mathrm{eq}}(z) \frac{1}{\sqrt{4 \pi D_{i}(z) \Delta t}} e^{-\frac{\Delta r_{i}^{2}}{4 D_{i}(z) \Delta t}},
$$

against the Gibbs-Boltzmann distribution. As shown in Figs. 4(b) and 4(c), Eq. (6) captures the early data very well. At long times, Eq. (6) remains valid only for $P_{x}$ and $P_{y}$. Nevertheless, the equilibrium regime being reached, $P_{z}$ can eventually be written as

$$
\lim _{\Delta t \rightarrow \infty} P_{z}(\Delta z)=\int_{0}^{\infty} d z P_{\mathrm{eq}}(z+\Delta z) P_{\mathrm{eq}}(z),
$$

which contains in particular the second moment:

$$
\lim _{\Delta t \rightarrow \infty}\left\langle\Delta z^{2}\right\rangle=\int_{-\infty}^{+\infty} d \Delta z \Delta z^{2} \int_{0}^{\infty} d z P_{\mathrm{eq}}(z+\Delta z) P_{\mathrm{eq}}(z) .
$$


As shown in Fig. 4(d), Eq. (8) captures the long-term data along $z$ very well.

We now wish to go beyond the previous average $\left\langle D_{i}\right\rangle$ of Eq. (5), and resolve the local diffusion coefficient $D_{i}(z)$. To measure local viscosities from experimental trajectories, a binning method is generally employed [35]. Although this technique is well suited for drift measurements, it suffers from a lack of convergence and precision when second moments or local diffusion coefficients have to be extracted [23]. In particular, the binning method did not allow us to measure specifically the local diffusion coefficient in the key interfacial region corresponding to $z<100 \mathrm{~nm}$. Additionally, Frishman and Ronceray have recently developed a robust numerical method using stochastic force inference, in order to evaluate spatially varying force fields and diffusion coefficients, from the information contained within the trajectories [23]. In practice, this is done by projecting the diffusion tensor onto a finite set of basis functions. We implemented this method, using fourth-order polynomials in our case. It allowed us to infer the local diffusion coefficients $D_{i}(z)$, down to $z=10$ $\mathrm{nm}$, as shown in Fig. 1(a). The results are in excellent agreement with the theoretical predictions, $D_{\|}(z)=D_{0} \eta / \eta_{\|}(z)$ and $D_{z}(z)=D_{0} \eta / \eta_{z}(z)$, using the effective viscosities of Eqs. (3) and (4), thus validating the method.

So far, through Figs. 1(a), 3(b), and 4, we have successively presented the various measured statistical quantities of interest, as well as their fits to corresponding theoretical models. Therein, we have essentially three free physical parameters, $B, \ell_{\mathrm{B}}$, and $\ell_{\mathrm{D}}$, describing the particle and its environment, as well as the a priori undetermined location of the $z=0$ origin. These four parameters are actually redundant among the various theoretical models. Therefore, in order to measure them accurately, we in fact perform all the fits simultaneously, using a Broyden-Fletcher-Goldfarb-Shanno algorithm that is well suited for unconstrained nonlinear optimization [36]. To do so, we construct a global minimizer:

$$
\chi^{2}=\sum_{n=1}^{N} \chi_{n}^{2}
$$

where we introduce the minimizer $\chi_{n}^{2}$ of each set $n$ among the $N$ sets of data, defined as

$$
\chi_{n}^{2}=\sum_{i=1}^{M_{n}} \frac{\left[y_{n i}-f_{n}\left(x_{n i}, \mathbf{b}\right)\right]^{2}}{f_{n}\left(x_{n i}, \mathbf{b}\right)^{2}},
$$

with $\left\{x_{n i}, y_{n i}\right\}$ the experimental data of set $n, M_{n}$ the number of experimental data points for set $n, f_{n}$ the model for set $n$, and $\mathbf{b}=\left(b_{1}, b_{2}, \ldots, b_{p}\right)$ the $p$ free parameters. In our case, $p=$ 4 , and $\left\{x_{n i}, y_{n i}\right\}$ represent all the experimental data shown in Figs. 1(a), 3(b), and 4.

Due to strong dependence of the normal diffusion coefficient $D_{z}$ with $z$, it is possible to find the wall position with a $10-n m$ resolution, thus overcoming a drawback of the Lorenz-Mie technique which only provides the axial distance relative to the focus of the objective lens. Additionally, the three physical parameters globally extracted from the multifitting procedure are $B=4.8 \pm 0.6, \ell_{\mathrm{D}}=21 \pm 1 \mathrm{~nm}$, and $\ell_{\mathrm{B}}=$ $530 \pm 2 \mathrm{~nm}$. Using the particle radius $a=1.518 \pm 0.006 \mu \mathrm{m}$ calibrated from the preliminary fits of the interference patterns to the Lorenz-Mie scattering function [see Figs. 2(c) and 2(d)], and the $1050 \mathrm{~kg} \mathrm{~m}^{-3}$ tabulated bulk density of polystyrene, we would have expected $\ell_{\mathrm{B}}=559 \mathrm{~nm}$ instead, which corresponds to less than $2 \%$ error, and might be attributed to nanometric offsets, such as, e.g., the particle and/or wall rugosities.

Finally, we investigate the total conservative force $F_{z}(z)$ acting on the particle along $z$. By averaging the overdamped Langevin equation over a fine-enough $z$-binning grid and short enough time interval $\Delta t$, one gets the Itō convention (corresponding to our definition of $\Delta z$ ):

$$
F_{z}(z)=6 \pi \eta_{z}(z) a \frac{\langle\Delta z\rangle}{\Delta t}-k_{\mathrm{B}} T \frac{D_{z}^{\prime}(z)}{D_{z}(z)},
$$

where the last term corresponds to the additional contribution due to the nontrivial integration of the multiplicative noise [20,37-39], with the prime denoting the derivative with respect to $z$. From the averaged measured vertical drifts $\langle\Delta z\rangle$, and invoking Eq. (4), one can reconstruct $F_{z}(z)$ from Eq. (11), as shown in Fig. 1(b). We stress that the statistical error on the force measurement is comparable to the thermal-noise limit [40]:

$$
\Delta F=\sqrt{24 \pi k_{\mathrm{B}} T \eta_{z}(z) a / \tau_{\mathrm{box}}(z)},
$$

where $\tau_{\text {box }}(z)$ is the total time spent by the particle in the corresponding box of the $z$-binning grid. To corroborate these measurements, we invoke Eq. (1) and express the total conservative force $F_{z}(z)=-U^{\prime}(z)$ acting on the particle along $z$ :

$$
F_{z}(z)=k_{\mathrm{B}} T\left(\frac{B}{\ell_{\mathrm{D}}} e^{-\frac{z}{\ell_{\mathrm{D}}}}-\frac{1}{\ell_{\mathrm{B}}}\right) .
$$

Using the physical parameters extracted from the above multifitting procedure, we plot Eq. (13) in Fig. 1(b). The agreement with the data is excellent, thus showing the robustness of the force measurement. In particular, we can measure forces down to a distance of $40 \mathrm{~nm}$ from the surface. Additionally, far from the wall, we are able to resolve the actual buoyant weight $F_{\mathrm{g}}=-7 \pm 4 \mathrm{fN}$ of the particle. This demonstrates that we reach the femtonewton resolution, and that this resolution is solely limited by thermal noise.

To conclude, we have successfully built a multiscale statistical analysis for the problem of freely diffusing individual colloids near a rigid wall. Combining the equilibrium distribution in position, time-dependent non-Gaussian statistics for the spatial displacements, a method to infer local diffusion coefficients, and a multifitting procedure allowed us to reduce drastically the measurement uncertainties and reach the nanoscale and thermal-noise-limited femtonewton spatial and force resolutions, respectively. The ability to measure tiny surface forces, locally, and at equilibrium, as well as the possible extension of the method to nonconservative forces and out-of-equilibrium settings [41,42], opens fascinating perspectives for nanophysics and biophysics.

We thank Elodie Millan, Louis Bellando de Castro, Julien Burgin, Bernard Trégon, Abdelhamid Maali, David Dean, and Mathias Perrin for interesting discussions. We acknowledge funding from the Bordeaux IdEx program LAPHIA (Grant No. ANR-10IDEX-03-02), Arts et Science (Sonotact 
Grant No. 2017-2018), Région Nouvelle Aquitaine (Grant No. 2018-1R50304), and from the Agence Nationale de la Recherche (Grant No. ANR-21-ERCC-0010-01 EMetBrown).
[1] A. Einstein, Über die von der molekularkinetischen theorie der wärme geforderte bewegung von in ruhenden flüssigkeiten suspendierten teilchen, Ann. Phys. 322, 549 (1905).

[2] J. Perrin, Les Atomes (Flammarion, Paris, 2014).

[3] B. U. Felderhof, Effect of the wall on the velocity autocorrelation function and long-time tail of Brownian motion, J. Phys. Chem. B 109, 21406 (2005).

[4] B. Wang, S. M. Anthony, S. C. Bae, and S. Granick, Anomalous yet Brownian, Proc. Natl. Acad. Sci. USA 106, 15160 (2009).

[5] A. V. Chechkin, F. Seno, R. Metzler, and I. M. Sokolov, Brownian yet Non-Gaussian Diffusion: From Superstatistics to Subordination of Diffusing Diffusivities, Phys. Rev. X 7 , 021002 (2017).

[6] C. I. Bouzigues, P. Tabeling, and L. Bocquet, Nanofluidics in the Debye Layer at Hydrophilic and Hydrophobic Surfaces, Phys. Rev. Lett. 101, 114503 (2008).

[7] L. Joly, C. Ybert, and L. Bocquet, Probing the Nanohydrodynamics at Liquid-Solid Interfaces Using Thermal Motion, Phys. Rev. Lett. 96, 046101 (2006).

[8] J. Mo, A. Simha, and M. G. Raizen, Brownian motion as a new probe of wettability, J. Chem. Phys. 146, 134707 (2017).

[9] E. R. Dufresne, T. M. Squires, M. P. Brenner, and D. G. Grier, Hydrodynamic Coupling of Two Brownian Spheres to a Planar Surface, Phys. Rev. Lett. 85, 3317 (2000).

[10] L. P. Faucheux and A. J. Libchaber, Confined Brownian motion, Phys. Rev. E 49, 5158 (1994).

[11] E. R. Dufresne, D. Altman, and D. G. Grier, Brownian dynamics of a sphere between parallel walls, Europhys. Lett. 53, 264 (2001).

[12] R. Lopez-Fernandez, M. D. Carbajal-Tinoco, and J. L. ArauzLara, Asymmetry in Colloidal Diffusion Near a Rigid Wall, Phys. Rev. Lett. 99, 138303 (2007).

[13] H. B. Eral, J. M. Oh, D. van den Ende, F. Mugele, and M. H. G. Duits, Anisotropic and hindered diffusion of colloidal particles in a closed cylinder, Langmuir 26, 16722 (2010).

[14] P. Sharma, S. Ghosh, and S. Bhattacharya, A high-precision study of hindered diffusion near a wall, Appl. Phys. Lett. 97, 104101 (2010).

[15] J. Mo, A. Simha, and M. G. Raizen, Broadband boundary effects on Brownian motion, Phys. Rev. E 92, 062106 (2015).

[16] M. Matse, M. V. Chubynsky, and J. Bechhoefer, Test of the diffusing-diffusivity mechanism using near-wall colloidal dynamics, Phys. Rev. E 96, 042604 (2017).

[17] D. C. Prieve, Measurement of colloidal forces with TIRM, Adv. Colloid Interface Sci. 82, 93 (1999).

[18] A. Banerjee and K. D. Kihm, Experimental verification of near-wall hindered diffusion for the Brownian motion of nanoparticles using evanescent wave microscopy, Phys. Rev. E 72, 042101 (2005).

[19] S. K. Sainis, V. Germain, and E. R. Dufresne, Statistics of Particle Trajectories at Short Time Intervals Reveal fN-Scale Colloidal Forces, Phys. Rev. Lett. 99, 018303 (2007).
[20] G. Volpe, L. Helden, T. Brettschneider, J. Wehr, and C. Bechinger, Influence of Noise on Force Measurements, Phys. Rev. Lett. 104, 170602 (2010).

[21] W. Wang, J. S. Guasto, and P. Huang, Measurement bias in evanescent wave nano-velocimetry due to tracer size variations, Exp. Fluids 51, 1685 (2011).

[22] M. Li, O. Sentissi, S. Azzini, G. Schnoering, A. CanaguierDurand, and C. Genet, Subfemtonewton force fields measured with ergodic Brownian ensembles, Phys. Rev. A 100, 063816 (2019).

[23] A. Frishman and P. Ronceray, Learning Force Fields from Stochastic Trajectories, Phys. Rev. X 10, 021009 (2020).

[24] C. F. Bohren and D. R. Huffman, Absorption and Scattering of Light by Small Particles (Wiley, New York, 1998).

[25] M. Mishchenko, Scattering, Absorption, and Emission of Light by Small Particles (Cambridge University, Cambridge, England, 2002).

[26] S.-H. Lee, Y. Roichman, G.-R. Yi, S.-H. Kim, S.-M. Yang, A. van Blaaderen, P. van Oostrum, and D. G. Grier, Characterizing and tracking single colloidal particles with video holographic microscopy, Opt. Express 15, 18275 (2007).

[27] P. D. J. van Oostrum, Using light scattering to track, characterize and manipulate colloids, Ph.D. thesis, University of Amsterdam, 2011.

[28] B. J. Krishnatreya, Precision measurements of colloidal interactions and dynamics, Ph.D. thesis, New York University, 2014.

[29] J. Israelachvili, Intermolecular and Surface Forces (Academic, New York, 2011).

[30] M. R. Oberholzer, N. J. Wagner, and A. M. Lenhoff, Grand canonical Brownian dynamics simulation of colloidal adsorption, J. Chem. Phys. 107, 9157 (1997).

[31] H. Brenner, The slow motion of a sphere through a viscous fluid towards a plane surface, Chem. Eng. Sci. 16, 242 (1961).

[32] M. A. Bevan and D. C. Prieve, Hindered diffusion of colloidal particles very near to a wall: Revisited, J. Chem. Phys. 113, 1228 (2000).

[33] M. V. Chubynsky and G. W. Slater, Diffusing Diffusivity: A Model for Anomalous, yet Brownian, Diffusion, Phys. Rev. Lett. 113, 098302 (2014).

[34] S. Hapca, J. W. Crawford, and I. M. Young, Anomalous diffusion of heterogeneous populations characterized by normal diffusion at the individual level, J. R. Soc., Interface 6, 111 (2009).

[35] R. Friedrich, J. Peinke, M. Sahimi, and M. Reza Rahimi Tabar, Approaching complexity by stochastic methods: From biological systems to turbulence, Phys. Rep. 506, 87 (2011).

[36] Y.-H. Dai, Convergence properties of the BFGS algorithm, SIAM J. Optim. 13, 693 (2002).

[37] R. Mannella and P. V. E. McClintock, Comment on "Influence of Noise on Force Measurements," Phys. Rev. Lett. 107, 078901 (2011).

[38] G. Volpe, L. Helden, T. Brettschneider, Jan Wehr, and C. Bechinger, Reply to Mannella and McClintock, Phys. Rev. Lett. 107, 078902 (2011). 
[39] R. Mannella and P. V. E. McClintock, Ito versus Stratonovich: 30 years later, Fluct. Noise Lett. 11, 1240010 (2012).

[40] L. Liu, S. Kheifets, V. Ginis, and F. Capasso, Subfemtonewton Force Spectroscopy at the Thermal Limit in Liquids, Phys. Rev. Lett. 116, 228001 (2016).

[41] Y. Amarouchene, M. Mangeat, B. V. Montes, L. Ondic, T. Guérin, D. S. Dean, and Y. Louyer, Nonequilibrium Dynamics
Induced by Scattering Forces for Optically Trapped Nanoparticles in Strongly Inertial Regimes, Phys. Rev. Lett. 122, 183901 (2019).

[42] M. Mangeat, Y. Amarouchene, Y. Louyer, T. Guérin, and D. S. Dean, Role of nonconservative scattering forces and damping on Brownian particles in optical traps, Phys. Rev. E 99, 052107 (2019). 